\title{
Proceedings of 2018 Asia Pacific Medicine and Bio- Immunology Association (APAMBI)
}

\author{
Standing Committee: Huasong Zeng", Banfuwu Wü, Jiangying Yang ${ }^{\#}$, Jianchuan Xia*, Fusheng Wang*, Xiaobing Fu* \\ "Co first authors \\ *Common corresponding authors
}

\section{First Class Outstanding Paper Award}

A randomized double-blind controlled clinical trial to evaluate the efficacy of recombinant human tumor necrosis factor- $\alpha$ receptor II fusion protein in juvenile idiopathic arthritis

\section{Zeng Ping, Zeng Hua-song*}

Women and Children's Medical Center, Guangzhou Medical University, China

\section{Correspondence: Zeng Hua-song}

Objective: Research the Recombinant Human Tumor Necrosis Factor- $\alpha$ Receptor type-II fusion protein antibody (Etanercept) for Treatment of juvenile idiopathic arthritis (JIA). A randomized doubleblind controlled clinical trial was conducted, to provide evidence to better use of this drug in clinical application.

Methods: Randomized double-blind principle was applied to divide the 124 JIA cases into control and treatment group. Slow-acting antirheumatic, nonsteroidal drugs or adrenocortical hormone were applied in these cases as basic drugs. There are no significant differences $(\mathrm{P}>0.05)$ of clinical classification and basic treatment between the two groups. 62 cases of treatment group achieved subcutaneous Etanercept, $0.8 \mathrm{mg} / \mathrm{kg}$ per week with a six mouth's period of treatment. The group included 17 oligoarticular cases (27.4 2\%), 15 polyarticular cases (24.19\%) and 30 SO-JIA cases (48.38\%). The ACR Ped - 30, 50,70 were used to assess the clinical efficacy. The occurrence of adverse reactions is monitored and recorded at the same time.

Results: The remission rate of different cases in treatment group was different at each time point $(\mathrm{P}<0.05)$. The clinical remission rate of SO-JIA cases was lower compared with the two other types $(\mathrm{P}<0.05)$. The SO-JIA cases achieved $44 \%$ ACR Pedi50 remission after 3 months' treatment, and after 6 months' treatment they received $40.7 \%$ ACR Pedi50 remission and 29.6\%ACR Pedi70 remission. The oligoarticular cases and polyarticular cases didn't have obvious difference in ACR Pedi30, 50, 70 remission rates at different time. $80 \%$ of these cases had ACR Pedi50 remission after 6 months' treatment and above $50 \%$ had ACR Pedi70 remission. These were significantly different $(\mathrm{P}<0.05)$ compared with the control group. In the SO-JIA treatment group, Etanercept was effective in 3 SOJIA-MAS cases that conventional treatment didn't help, and this did not conform to the report in the literature. 2 So - JIA cases merged with muscular stiffness after 1-week treatment. 5 So-JIA cases had upper respiratory tract infection, diarrhoea and other infection for several times during the treatment, one of them had chickenpox infection. They all got remission after drug withdrawal and symptomatic treatment. The adverse reactions of
SO-JIA subgroups were $23.3 \%$, while the other 2 types had no adverse reaction.

Conclusion: Etanercept has a good therapeutic effect for JIA cases that mainly have articular lesions such as the oligoarticular type and polyarticular type. The adverse reactions of short term (within 6 months) are rare, the long-term security and stability of joint recovery are subject to a large sample, multi-center long-term followup observational study. Etanercept can make So-JIA patient's clinical remission, but infection and other adverse events should be strictly guarded against. We can consider Etanercept for the SO-JIA patients that the conventional treatment is of no effect or SO-JIA patients merge with MAS. But some of patients applied with Etanercept may get worse, there are no reliable predictor for this by now. The Long-term efficacy and adverse reaction require further observation.

Keywords: recombinant human tumor necrosis factor- $\alpha$ receptor; type-II fusion protein antibody, juvenile idiopathic arthritis

\section{Second Class Outstanding Paper Awards}

Study on the expression level of myeloid source suppressor cells in juvenile idiopathic arthritis and the mechanism of the effect on the differentiation of TH17 cells

\section{Suyun Cheng, Huasong Zeng}

Women and Children's Medical Center, Guangzhou Medical University, China

Objective: Myeloid-derived suppressor cells(MDSCs)have the potential to suppress autoimmune $\mathrm{T}$ cell response, while the levels and function of it had not been studied in juvenile idiopathic arthritis (JIA), its role in type 17 helper cell differentiation and the pathogenesis of autoimmune arthritis has not been determined. So, our aims are to investigate the frequency of MDSCs in peripheral blood of JIA and analysed its correlation with cytokines, providing clinical basis for the generation and function of MDSCs in JIA; investigate the influence and mechanism of MDSCs on Th17 cell differentiation in collagen induced arthritis (CIA) mice, to clarify the pathogenesis of autoimmune arthritis and find new therapeutic targets for autoimmune arthritis.

Methods: A total of 34 patients with JIA, disease activity was evaluated by calculating the 28 -joint Disease Activity Score (DAS28)

Received: January 15, 2019; Accepted: January 22, 2019; Published: January 25, 2019 
based on clinical information. JIA patients were divided into active group and inactive group according to the DAS28 score. 20 healthy controls were recruited from the Health Care Section of our hospital. The frequency of MDSC in peripheral blood from JIA patients and healthy controls were determined by flow cytometry; ELISA was used to detect plasma IL-1 $\beta$, IL-6, IL-10 and IL-17A of JIA patients and healthy controls; RT-qPCR was performed to detect the expression levels of STAT3, IL-17A gene in patients with JIA and control subjects, and Spearman correlation was used to investigate the association between MDSC and DAS28 score, cytokines.

(2) The frequency of MDSCs in Splenic mononuclear cells and the levels of pathogenic T helper 17 cells were examined in arthritic mice by flow cytometry, ELISA was used to detect plasma IL-1 $\beta$, IL-10 and IL-17A of CIA and Normal mice; The MDSCs in arthritic mice were also examined for their $\mathrm{T}$ cell suppressive activity and the capacity of pro-Th17 cell differentiation. The mechanism of MDSCs pro-Th17 cell differentiation was determined by IL- $1 \beta \mathrm{mAb}$ or IL-1ra in coculturing MDSCs with CD4+T cells under Th17-polarising conditions.

Results: (1)The frequency of MDSC, and the levels of plasma cytokines IL-1 $\beta$, IL-6, IL-10 increased significantly in JIA patients compared with healthy control group, while STAT3,IL-17A mRNA had no differences; The frequency of MDSC correlated positively with disease activity,IL-1 $\beta$ and IL-10; negatively correlated with the STAT3 mRNA; but not correlated with the IL-6, IL-17A by Spearman's test.

(2) MDSCs significantly expanded in CIA, which correlated positively with Th17 cells. While displaying T cell suppressive activity, MDSCs promoted Th17 cells polarisation ex vivo under Th17polarising conditions, indicated by significantly increased number of Th17 cells ,elevation of IL-17A production, and upregulation of the STAT3 and ROR $\gamma$ t. Mechanistic studies show that IL- $1 \beta$ represents a major mediator of MDSCs-facilitated Th17 differentiation .

Conclusion: Our studies show that MDSCs have the capacity to pro inflammatory in autoimmune arthritis and can drive Th17 cell differentiation depend on IL- $1 \beta$, may be a critical pathogenic factor in autoimmune arthritis.

Keywords: Myeloid-derived suppressor cells; Juvenile idiopathic arthritis; IL-1 $\beta$; Th17 cells; Collagen induced arthritis; Rheumatoid arthritis

Capecitabine inhibits multiple myeloma growth in MPC-11 cell- burdened mice by regulating expressions of miRNAs

Han Hong ${ }^{1, *}$, Yang Fen ${ }^{1}$, Cai Wen ${ }^{1}$, Chen Hui ${ }^{1}$, Zeng Yan ${ }^{1}$, Yan Yijin ${ }^{2}$

${ }^{1}$ Department of Hematology of Liyuan hospital of Tongji Medical College, Huazhong University of Science and Technology, People's Republic of China

${ }^{2}$ The first clinic Institute of Tongji Medical College, Huazhong University of Science and Technology

\section{Correspondence: Han Hong}

Aim: To investigate the effects of Capecitabine (CAP) on multiple myeloma(MM) MPC-11 cells burdened mice and its mechanisms.

Methods: MPC-11 cells were injected subcutaneously in the right axillary cavity of mice to establish MM-burdened model, the antimyeloma effect of CAP was evaluated using stomach perfusion in vivo. MPC-11 cells were treated with different doses of CAP for $48 \mathrm{~h}$ in vitro, cell viability was accessed by CCK-8; cell apoptosis was determined by flow cytometry; gene-chip microarray was performed to assess the changes of miRNAs in MPC-11 cells after treatment with IC50 dose of CAP; MiRNA expressions were further evaluated by real-time quantitative PCR(RQ-PCR).

Results: After two weeks of continuous treatment, mice were euthanized, and the myeloma tissues were weight. Compared with the control group, CAP has significantly inhibited the growth of myeloma in mice $(\mathrm{P}<0.01)$. In addition, CAP could inhibit cell viability and promote cell apoptosis in a dose-dependent manner. Gene-chip analysis indicated that 84 genes were significantly up-regulated, whereas 24 genes were significantly down-regulated. The expressions of miR-29b and miR-15a increased over 1.5 -fold, whereas the expression of miR21 was down-expressed over $60 \%$ as determined by RQ-PCR analysis.

Conclusions: CAP has a significant anti-myeloma effects, and its underlying mechanism might be inhibiting cell viability, promoting cell apoptosis and regulating related miRNAs genes expression.

Keywords: CAP; MPC-11 cell-burdened mice; multiple myeloma growth; viability; apoptosis; miRNA

\section{Third Class Outstanding Paper Awards}

Study on the anti-tumor effect of CTL mediated by double specific antibody in positive pancreatic cancer with targeted KIF20A

Yuan Jia, Xiaoling Yang, Huijing Feng, Pengmin Liu, Junping Zhang

Laboratory of Oncology Medicine / Biotherapy Center of Shanxi DAYI Hospital 030032, Taiyuan, Shanxi China

Background: Pancreatic cancer is a malignant tumor with a high mortality rate. It has a survival period of only about $5 \%$ for five years and has a poor prognosis. Surgery is currently an effective method to extend survival, but the proportion of surgical patients with pancreatic cancer is only $9-22 \%$. Therefore, there is an urgent need to explore a more effective treatment.

Objective: At present, many basic and clinical studies on CTL treatment of pancreatic cancer are being carried out, showing that specific $\mathrm{T}$ lymphocytes have far-reaching and broad application prospects. The screening of tumor antigens for specific CTL induction has also become a hot topic in the academic community. As a tumorrelated antigen, KIF20A (mitotic kinesin 2) is highly expressed in pancreatic cancer cells but not expressed in normal cells (or low expression), and immunotherapy (CTL) for pancreatic cancer has great potential.

Methods: Bi20A, which was targeted at KIF20A, was prepared by antibody reduction method and loaded on VC sensitive DC-T cells, which effectively improved the killing efficiency of $\mathrm{T}$ cells against KIF20A positive pancreatic adenocarcinoma cells.

Results: Microscopic observations showed that DC-T cells loaded with Bi20A bispecific antibodies could spontaneously search for tumor cells and actively accumulate to KIF20A positive pancreatic cancer cells under the ADCC effect. At the same time, they showed good tumor killing activity. The 12-hour tumor cell killing activity is nearly $100 \%$ (target ratio 30:1).

Conclusion: The $\mathrm{T}$ cell effect mediated by bispecific antibody can effectively kill tumor cells and has good spontaneous targeting ability. Good clinical application prospect.

Keywords: Pancreatic cancer; immunotherapy; targeted KIF20A

The results are as follows: 


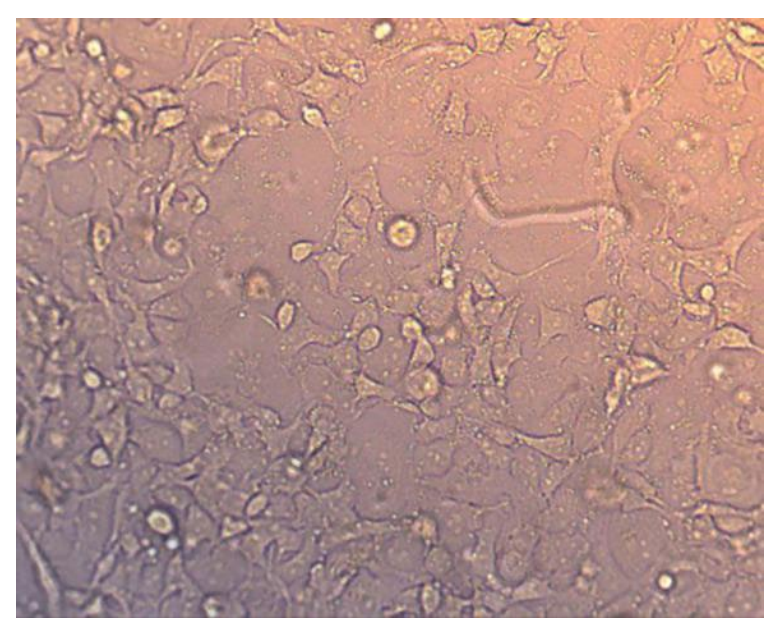

Figure 1. Microscopically observed wall growth of KIF20A positive pancreatic cancer cells(X 200 times)

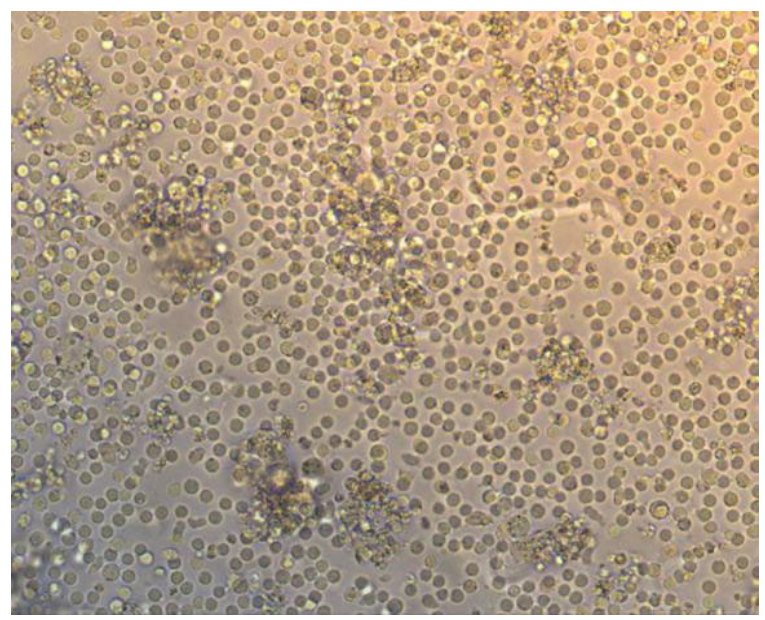

Figure 2. The effect of T cells without Bi20A bispecific antibodies on KIF20A + tumor cells(4h) shows that lymphocytes(T cells) are evenly distributed in the field of view, and only some $\mathrm{T}$ cells are clustered around tumor cells. T cell tropism.

Target ratio 30:1

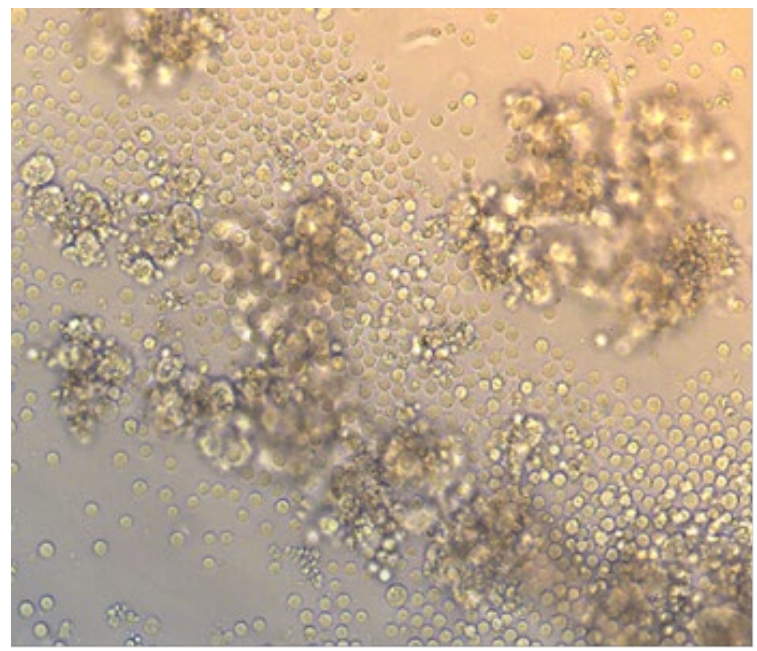

Figure 3. The T cells loaded with Bi20A bispecific antibodies showed good tropism and killing activity to tumor cells. A large number of $\mathrm{T}$ cells were gathered around the tumor cells, and the concentrations were different. There were a large number of $\mathrm{T}$ cells around the tumor cells. Gathering, tumor cells were killed(4h)

Target ratio 30:1

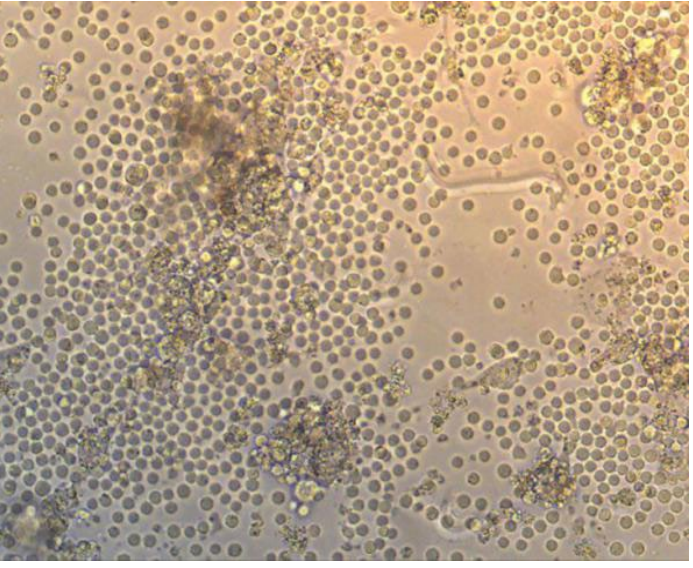

Figure 4. T-cells loaded with Bi20A bispecific antibodies accumulate in large numbers around tumor cells and kill tumor cells. (4h)

Target ratio 30:1

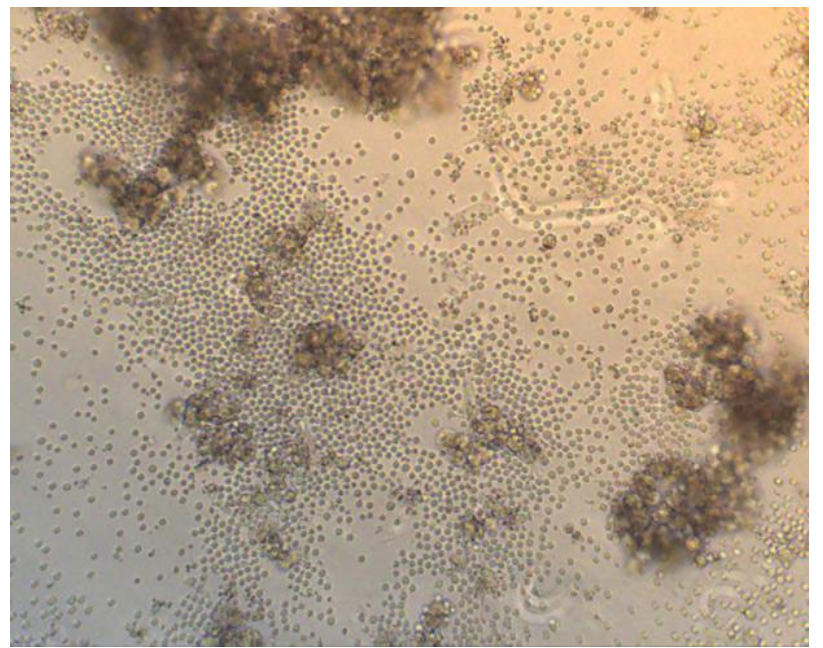

Figure 5. U-cells loaded with $\mathrm{Bi} 20 \mathrm{~A}$ bispecific antibodies have good targeting and are concentrated around tumor cells using the ADCC effect.

Target ratio 30:1

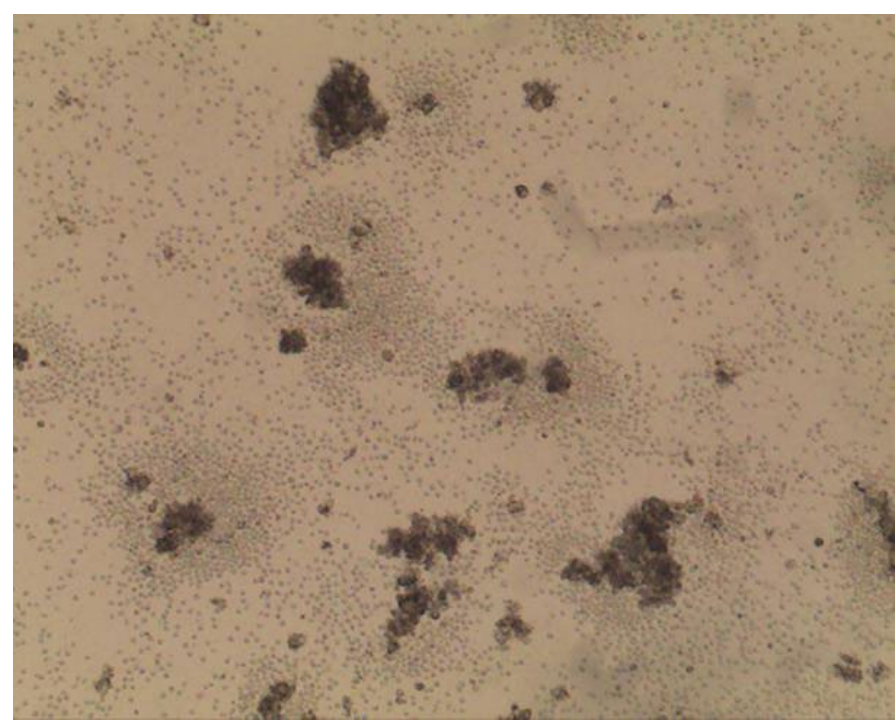

Figure 6. T-cells loaded with Bi20A bispecific antibodies have good targeting and are concentrated around tumor cells using the ADCC effect.

Target ratio 30:1 


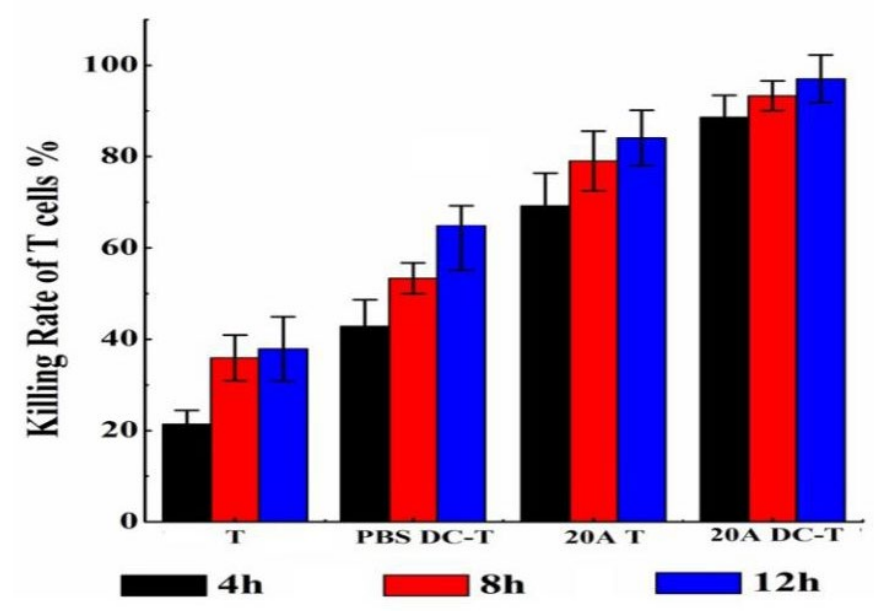

Figure 7. The time gradient results show that the T cells loaded with Bi20A bispecific antibodies have a significantly higher ability to kill tumor cells than unloaded T cells. At the same time, the results also showed that the cells cultured by DC-T mixed culture showed a significant CTL effect after the Bi20A bispecific antibody was loaded, and the targeting effect was stronger.

Target ratio 30:1

Safety of DCVAC/LuCa combined with chemotherapy for treating advanced non-small cell lung cancer

Cao Shu-hui, Zhou yan, LI Jing-wen, Zhong Hua

Department of Respiratory, Shanghai Chest Hospital, Shanghai Jiao Tong University, Shanghai 200030, China

Objective: To evaluate the safety of DCVAC/LuCa combined with pemetrexed/carboplatin in patients with stage IV non-small cell lung cancer (NSCLC).

Methods: A total of 20 newly diagnosed stage IV, non-squamous, wild-type epidermal growth factor receptor (EGFR), anaplastic lymphoma kinase (ALK) negative or unknown NSCLC patients were randomized into test group $(n=10)$ and control group $(n=10)$. Test group received pemetrexed/ carboplatin chemotherapy (4-6 cycles), combined with DCVAC/LuCa treatment in the third cycle, followed pemetrexed monotherapy in combination with DCVAC/LuCa; control group received pemetrexed/carboplatin chemotherapy (4-6 cycles), followed pemetrexed alone.

Results: The efficiency of the patients in two groups were evaluated and adverse effects (AEs) were collected and analysed. There was a significant positive correlation between $\mathrm{DCVAC} / \mathrm{LuCa}$ transfusion dose and progression-free survival (PFS) (r0.773, P0.009). The common AEs in both groups were chemotherapy related leukopenia, hemoglobin decrease etc. All AEs were grade 1 or 2 according to common terminology criteria for adverse events (CTCAE) V4.03, and there were no grade 4 toxicities or treatment-related deaths. One patient in test group got non-infectious fever and body ache and returned to normal without treatment.

Conclusion: In patients with stage IV NSCLC, DCVAC/LuCa therapy is well tolerated with the favourable safety. safety
Lipoxin A4 and Nuclear factor kappa B in serum and urine: potential markers for Paediatric Henoch-Schönlein purpura Nephritis

Mengjiao Shen ${ }^{1}$, Qianqian Ying ${ }^{1}$, Yunyan $\mathrm{Li}^{2}$, Ling $\mathrm{Wu}^{2}$, Yazhen $\mathrm{Di}^{2}$, Jiapei Wang ${ }^{2}$, Shiwei Fu ${ }^{1}$

${ }^{1}$ Medicine School of Ningbo University, 818 Fenghua Road, Ningbo, Zhejiang 315000, China

${ }^{2}$ Department of Pediatric Rheumatoid Immunity, Ningbo Women and Children's Hospital, 339 Liuting Street, Ningbo, Zhejiang 315000, China

Objective: The study was conducted to observe the serum and urine levels of Lipoxin A4 (LXA4) and Nuclear Factor-kappa B(NF-кB) in pediatric Henoch-Schönlein purpura (HSP), and their association with HSP.

Methods: 99 HSP cases and 27 healthy controls (Controls) were recruited in this study. The cases were further divided into 3 groups based on the clinical features: Group 1 (37 HSP without gastrointestinal (GI) involvement and nephritis), Group 2 (30 HSP with GI involvement but without nephritis) and Group 3 (32 HSP with nephritis (HSPN)). LXA4 and NF- $\mathrm{kB}$ levels in each individual's serum and urine specimens were estimated by enzyme-linked immunosorbent assay (ELISA).

Results: Serum and urine LXA4 levels in Group1 and Group2 were significantly higher than Controls $(\mathrm{P}$ all $<0.05)$. And LXA4 levels in Group3 were significantly lower compared with Group1 and Group2 (P all<0.05). Differently, NF- $\kappa B$ levels in serum and urine in Group1, Group2 and Group3 were all markedly higher compared with Controls $(\mathrm{P}$ all<0.05), NF- $\mathrm{kB}$ levels in Group3 were significantly higher than Group1 and Group2 (P all $<0.05)$. Serum levels of LXA4 and NF- $\kappa B$ in HSP were significantly higher compared with urine $(\mathrm{P}$ both $<0.01)$. Additionally, serum and urine LXA4 levels in HSP were negatively associated with 24-hour proteinuria and urinary $\mathrm{mAlb}$

$(\mathrm{P}$ all $<0.01), \mathrm{NF}-\kappa \mathrm{B}$ levels were positively associated with 24 -hour proteinuria, urinary $\mathrm{mAlb}$ and $\mathrm{D}$-dimer $(\mathrm{P}$ all $<0.05)$. Above all, there was a negative correlation between LXA4 and NF- $\kappa B$ in HSP $(\mathrm{P}<0.05)$.

Conclusions: This study supports that low LXA4 and high NF-kB levels could be potential risk markers for HSPN. And urine examination could be used in clinical practice extensively.

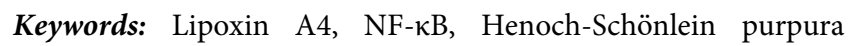
Nephritis, serum, urine, pediatric

\section{Poster Paper Awards.}

Elevated interleukin-1 $\beta$ and interleukin- 6 levels in the serum of children with hyperuricemia

Yazhen $\mathrm{Di}^{1}$, Jiapei Wang ${ }^{1,2}$, Yuanling Chen ${ }^{1}, \mathrm{Nan} \mathrm{Sun}^{3}$, Ling $\mathrm{Wu}^{1}$, Xiahua Dai ${ }^{1}$, Mengjiao Shen ${ }^{2}$, Qianqian Ying ${ }^{2}$, Shiwei $\mathrm{Fu}^{2}$

${ }^{1}$ Department of Pediatric Rheumatology and Immunology, Ningbo Women and Children's Hospital, 339 Liuting Street, Ningbo, Zhejiang 315000, China

${ }^{2}$ Medicine School, Ningbo University, 818 Fenghua Road, Ningbo, Zhejiang 315000, China

${ }^{3}$ Department of Pediatric Immunity, Tianjin Children's Hospital, 238 Longyan Road, Tianjin 300074, China 
Purposes: To investigate the serum levels and clinical significance of interleukin-1 $\beta$ (IL-1 $\beta$ ) and interleukin-6 (IL-6) in children with hyperuricemia (HUA).

Methods: We included 71 children with HUA and 71 healthy children as controls. Children with HUA were divided into Group I and Group II according to whether they had a history of acute gout-like attacks (including sudden mono arthritis of rapid onset with intense pain and swelling) or not. Group I was examined twice (A, in the acute phase; $B$, in the remission phase). Serum IL- $1 \beta$ and IL- 6 levels were measured by enzyme-linked immunosorbent assay (ELISA).

Results: Serum IL-1 $\beta$ and IL-6 levels were increased in children with HUA and were overall statistically different from the control group $(\mathrm{P}<0.05$, respectively). Serum IL- $1 \beta$ and IL- 6 were significantly higher in Group IA in comparison with Group IB, Group II and controls ( $\mathrm{p}<0.05$, respectively) as well as in Group IB and Group II compared to controls $(\mathrm{p}<0.05$, respectively). In Group IB, the serum IL- $1 \beta$ and IL- 6 concentration were higher than Group II, but there were no statistical differences $(p>0.05)$. In addition, in children with HUA, serum IL- $1 \beta$ and IL- 6 levels were positively associated with white blood cell count (WBC), neutrophil count, monocyte count, uric acid levels, erythrocyte sedimentation rate (ESR), C Reactive Protein, Blood Urea Nitrogen and serum creatinine levels (all $p<0.05$ ), but were not associated with triglycerides, total cholesterol, LDL-C or HDL-C levels (all $\mathrm{p}>0.05$ ).

Conclusion: Elevated levels of serum IL-1 $\beta$ and IL- 6 were found in children with HUA and correlated with disease activity.

Keywords: Children; Hyperuricemia; IL-1 $\beta$; IL-6

Elevated urinary Monocyte Chemoattractant Protein-1 levels in children with Henoch-Schonlein purpura nephritis

Jiapei Wang1, Qianqian Ying ${ }^{2}$, Shiling Zhong ${ }^{1}$, Yuanling Chen ${ }^{1}$, Yazhen $\mathrm{Di}^{1}$, Xiahua Dai ${ }^{1}$, Jika Zheng ${ }^{1}$, Mengjiao Shen ${ }^{2}$

${ }^{1}$ Department of Pediatric Rheumatology and Immunology, Ningbo Women and Children's Hospital, 339 Liuting Street, Ningbo, Zhejiang 315000, China

${ }_{2}^{2}$ Medicine School, Ningbo University, 818 Fenghua Road, Ningbo, Zhejiang 315000, China

Purpose: To investigate the urinary monocyte chemoattractant protein-1 (MCP-1) levels and clinical significance in Henoch-Schonlein purpura (HSP) children with and without nephritis and determine the association of MCP-1 with proteinuria.

Methods: A total of 261 HSP children-with or without nephritisand 84 healthy control children were enrolled in this study. Of these, 126 HSP nephritis (HSPN) children were subdivided into three groups according to total urine protein in 24 hours (TUP): Group A, mild proteinuria group with TUP $<25 \mathrm{mg} / \mathrm{kg}$; Group B, moderate proteinuria group with TUP $\geq 25 \mathrm{mg} / \mathrm{kg}$ and $<50 \mathrm{mg} / \mathrm{kg}$; Group C, severe proteinuria group with TUP $\geq 50 \mathrm{mg} / \mathrm{kg}$. Urinary $\mathrm{MCP}-1$ levels were determined by ELISA. Levels of serum creatinine $(\mathrm{Cr})$, blood urea nitrogen (BUN), urinary a1-micro globulin ( 1 1-MG), micro-albumin (mAlb), immunoglobulin G (IgG), transferrin (TRF) and TUP were performed to determine their associations with MCP-1.

Results: Urinary MCP-1 was significantly higher in HSPN group in comparison with HSP group and controls $(\mathrm{P}<0.05)$, but no significant difference was found between the HSP group and the healthy group $(\mathrm{P}>0.05)$. The levels of urinary MCP-1 increased in parallel to the enhancement of total urine protein in 24 hours in HSPN patients. There were statistically significant differences among these three groups of HSPN children $(\mathrm{p}<0.05)$. Urinary MCP-1 correlated positively with urinary al-MG, mAlb, IgG, TRF and TUP in HSPN, whereas no correlation was observed with serum $\mathrm{Cr}$ and BUN.

Conclusions: MCP-1 was elevated in children with HSPN and correlated with proteinuria. Urinary MCP-1 could be used as a suitable, non-invasive biomarker to provide valuable information not only for the diagnosis of HSPN, but also for evaluation of severity of renal damage.

Keywords: Children; Henoch-Schonlein purpura nephritis; Monocyte chemoattractant protein-1; Proteinuria

Urate crystal deposition in hyperuricemia children: A dual energy CT study

Qianqian Ying', Jiapei Wang ${ }^{2}$, Yunyan $\mathrm{Li}^{2}$, Nan $\mathrm{Sun}^{3}$, Yazhen $\mathrm{Di}^{2}$, Mengjiao Shen ${ }^{1}$, Shiwei Fu${ }^{1}$

${ }^{1}$ Medicine School of Ningbo University, 818 Fenghua Road, Ningbo, Zhejiang 315000, China

${ }^{2}$ Department of Pediatric Rheumatoid Immunity, Ningbo Women and Children's Hospital, 339 Liuting Street, Ningbo, Zhejiang 315000, China

${ }^{3}$ Department of Pediatric Immunity, Tianjin Children's Hospital, 238 Longyan Road, Tianjin 300074, China

Objective: This study aimed to compare the frequency of dual energy CT(DECT) urate crystal deposition in children with hyperuricemia (HUA).

Methods: 42 HUA children were divided into Joint Group (n=27) and Asymptomatic Group ( $\mathrm{n}=15)$ according whether they had a history of arthritis symptom, including sudden mono arthritis of rapid onset with intense pain and swelling or not. We analysed DECT scans of feet from Joint Group and Asymptomatic Group.

Results: DECT urate deposits were observed in 25/27(92.6\%) children with symptomatic HUA, 9/15(60.0\%) with asymptomatic $\operatorname{HUA}(\mathrm{P}<0.05)$. We found 54 areas of urate deposition in Joint Group, DECT urate crystal deposition was more frequently observed in the first metatarsophalangeal (MTP) joint (25.9\%), ankle joint (14.8\%), and calcaneus (13.0\%). 24 urate deposits were found in Asymptomatic Group, DECT urate crystal deposition was more frequently observed in calcaneus (28.0\%), the first MTP joint (16.0\%), and the first metatarsal (16.0\%).

Conclusions: Urate deposition can occur in children with HUA, and these deposits occur more frequently in Joint Group.

Keywords: Hyperuricemia; Children; Dual-energy CT; Urate crystal; Joint injury

\section{Clinical analysis of 26 cases of children Behcet's disease}

Yazhen $\mathrm{Di}^{1}$, Ling $\mathrm{Wu}^{1}$, Xiahua Dai ${ }^{1}$, Yuanling Chen ${ }^{1}$, Yunyan $\mathrm{Li}^{1}$, Jiapei Wang ${ }^{1}$, Qianqian Ying ${ }^{2}$, Mengjiao Shen ${ }^{2}$, Shiwei $\mathbf{F u}^{2}$

${ }^{1}$ Department of Pediatric Rheumatology and Immunology, Ningbo Women and Children's Hospital, 339 Liuting Street, Ningbo, Zhejiang 315000, China

${ }^{2}$ Medicine School of Ningbo University, 818 Fenghua Road, Ningbo, Zhejiang 315000, China 
Purpose: To explore the clinical features of the pediatric Behcet's disease and strengthen the understanding of Behcet's disease (BD).

Method: 26 cases of the pediatric patients with $\mathrm{BD}$ who were diagnosed in hospital or out-patient clinic between April 1998 and December 2016 were selected, the course of illness, accompanying symptoms, and therapeutic effects of pediatric patients were recorded through outpatient follow-up or telephone follow-up, the initial clinical data and follow-up records were entered into the database, and such data was analysed and summarized.

Results: The age of onset is 1-15 years old; 0.5-10 years are required from onset to diagnosis, with a ratio of male to female as 8:5. In 26 cases (100\%), all initial symptoms were Recurrent Aphthous Ulcer (RAU). Other clinical symptoms occurred in the first and follow-up visit: 20 cases $(76.9 \%)$ were genital ulcers, 14 cases $(53.8 \%)$ were skin changes, 12 cases $(46.2 \%)$ were articular symptoms, 9 cases $(34.6 \%)$ were gastrointestinal symptoms, 6 cases (23.1\%) were ocular lesions, 5 cases $(19.2 \%)$ were perianal abscess, 5 cases $(19.2 \%)$ were neuropathy, 5 cases $(19.2 \%)$ were kidney involvement, 1 case (3.8\%) was cardiac lesions, and 18 cases were (69.2\%) positive in prick test. At the early stage of the disease, 20 cases $(76.9 \%)$ were treated at Department of Stomatology, 15 cases (57.7\%) were treated at Department of Urology, 10 cases $(38.5 \%)$ were treated at Department of Dermatology, 8 cases (30.8\%) were treated at Department of General Surgery, 6 cases $(23.1 \%)$ were treated at Department of Gastroenterology, 3 cases (11.5\%) were treated at Ophthalmology. After diagnosis, 7 cases (26.9\%) were performed with local therapy, 13 cases (50\%) were applied with single hormone therapy, 6 cases $(23.1 \%)$ were performed with hormoneimmunosuppressive therapy, 9 cases $(9 / 19,47.4 \%)$ were recurrent in case of hormone reduction or withdrawal.

Conclusion: RAU is the most common first symptom of Behcet's disease, which gradually involves multiple organ damage. The first visit of these pediatric patients is often performed at other departments, and all doctors should pay attention to the disease.

Keywords: Behcet's disease; Recurrent Aphthous Ulcer; clinical features

\section{Elevated interleukin-1 $\beta$ and interleukin- 6 levels in the serum of children with hyperuricemia}

Yazhen $\mathrm{Di}^{1}$, Jiapei Wang ${ }^{1,2}$, Yuanling Chen ${ }^{1}$, Nan Sun ${ }^{3}$, Ling $\mathrm{Wu}^{1}$, Xiahua Dai ${ }^{1}$, Mengjiao Shen ${ }^{2}$, Qianqian Ying ${ }^{2}$, Shiwei $\mathrm{Fu}^{2}$

${ }^{1}$ Department of Pediatric Rheumatology and Immunology, Ningbo Women and Children's Hospital, 339 Liuting Street, Ningbo, Zhejiang 315000, China

${ }^{2}$ Medicine School, Ningbo University, 818 Fenghua Road, Ningbo, Zhejiang 315000, China

${ }^{3}$ Department of Pediatric Immunity, Tianjin Children's Hospital, 238 Longyan Road, Tianjin 300074, China Department of Pediatric Rheumatoid Immunity, Ningbo Women and Children's Hospital, 339 Liuting Street, Ningbo, Zhejiang 315012, China

Objects: To investigate the serum levels and clinical significance of interleukin-1 $\beta$ (IL-1 $\beta$ ) and interleukin-6 (IL-6) in children with hyperuricemia (HUA).

Methods: We included 71 children with HUA and 71 healthy children as controls. Children with HUA were divided into Group I and Group II according to whether they had a history of acute gout-like attacks (including sudden monoarthritis of rapid onset with intense pain and swelling) or not. Group I was examined twice (A, in the acute phase; $B$, in the remission phase). Serum IL-1 $\beta$ and IL-6 levels were measured by enzyme-linked immunosorbent assay (ELISA).

Results: Serum IL-1 $\beta$ and IL-6 levels were increased in children with HUA and were overall statistically different from the control group $(\mathrm{P}<0.05$, respectively). Serum IL- $1 \beta$ and IL- 6 were significantly higher in Group IA in comparison with Group IB, Group II and controls ( $\mathrm{p}<0.05$, respectively) as well as in Group IB and Group II compared to controls $(\mathrm{p}<0.05$, respectively). In Group IB, the serum IL- $1 \beta$ and IL- 6 concentration were higher than Group II, but there were no statistical differences ( $p>0.05)$. In addition, in children with HUA, serum IL- $1 \beta$ and IL- 6 levels were positively associated with white blood cell count (WBC), neutrophil count, monocyte count, uric acid levels, erythrocyte sedimentation rate (ESR), C Reactive Protein, Blood Urea Nitrogen and serum creatinine levels (all $p<0.05$ ), but were not associated with triglycerides, total cholesterol, LDL-C or HDL-C levels (all $\mathrm{p}>0.05$ ).

Conclusion: Elevated levels of serum IL- $1 \beta$ and IL- 6 were found in children with HUA and correlated with disease activity.

Keywords: Children; Hyperuricemia; IL-1 $\beta$; IL-6

\section{Henoch Schonlein purpura in chinese children: Epidemiological and renal involvement of 936 cases}

Yazhen $\mathrm{Di}^{1}$, Shiling Zhong ${ }^{1}$, Ling $\mathrm{Wu}^{1}$, Jiapei Wang ${ }^{1}$, Mengjiao Shen ${ }^{2}$, Qianqian Ying ${ }^{2}$, Shiwei Fu

${ }^{1}$ Department of Pediatric Rheumatology and Immunology, Ningbo Women and Children's Hospital, 339 Liuting Street, Ningbo, Zhejiang 315000, China

${ }^{2}$ Medicine School of Ningbo University, 818 Fenghua Road, Ningbo, Zhejiang 315000, China

${ }^{3}$ Department of Pediatric Rheumatology and Immunology, Ningbo Women and Children's Hospital, 339 Liuting Street, Ningbo, Zhejiang 315000, China

Objective: To examine epidemiological, clinical, and renal involvement in Chinese children with Henoch Schonlein purpura (HSP).

Methods: Retrospective study of children discharged with a diagnosis of HSP from the Ningbo Women and Children's Hospital, between January 2013 and December 2015. Epidemiological, clinical, treatment, and outcome were collected by reviewing medical charts.

Results: 936 children entered the study, M: F = 1.2:1; mean age $6.90 \pm 2.63$ years. A majority of the cases were from rural areas in $81.9 \%$. At onset, purpura was present in all cases, gastrointestinal involvement in $37 \%$, arthritis/arthralgias in $31 \%$, renal involvement in $40.4 \%$, scrotal edema in $0.4 \%$. Their mean age at presentation of HSPN was $7.13 \pm 2.78$ years, which was significantly higher than the age of those without nephritis $(\mathrm{P}=0.031)$. Age at presentation, gastrointestinal involvement and joint involvement were the risk factors of renal involvement.

Conclusion: This study suggests that in children with HSP, patients with older than 10 years, clinically gastrointestinal, and joint involvement, may be predictive of renal involvement.

Keywords: Henoch Schonlein purpura; children; renal involvement 

Osteopontin may mediate the pathogenesis of juvenile
idiopathic arthritis

Yazhen $\mathrm{Di}^{1}$, Jiapei Wang ${ }^{1}$, Yuanling Chen ${ }^{1}$, Ling $\mathrm{Wu}^{1}$, Yunyan $\mathrm{Li}^{1}$, Hanchun $\mathrm{Yu}^{1}$, Mengjiao Shen ${ }^{2}$, Qianqian Ying ${ }^{2}$, Shiwei $\mathrm{Fu}^{2}$

${ }^{1}$ Department of Pediatric Rheumatology and Immunology, Ningbo Women and Children's Hospital, 339 Liuting Street, Ningbo, Zhejiang 315000, China

${ }^{2}$ Medicine School of Ningbo University, 818 Fenghua Road, Ningbo, Zhejiang 315000, China, Department of Pediatric Rheumatology and Immunology, Ningbo Women and Children's Hospital, 339 Liuting Street, Ningbo, Zhejiang 315000, China

Background: To evaluate the serum and synovial fluid expression of osteopontin (OPN) and its possible role in the pathogenesis of Juvenile idiopathic arthritis (JIA).

Methods: Fifty patients with juvenile idiopathic arthritis (JIA) were studied. Among them, 10 patients underwent synovial fluid (SF) aspiration before steroid injections. Serum samples monitored by all enrolled patients including 50 age-matched children with inguinal hernia surgery (control group). Serum and SF were measured by enzyme-linked immunosorbent assay (ELISA) for OPN.

Results: Serum OPN expression was increased in active JIA compared to inactive JIA and control group, with no significant differences among the different JIA subtypes. Levels of serum in OPN were significantly correlated with hemoglobin and platelet.

Conclusion: The level of OPN rises sharply in JIA active stage and JIA with joint damage. OPN may play an important role in JIA pathogenesis and the process of JIA with joint damage.

Keywords: Juvenile idiopathic arthritis; Osteopontin; Pathogenesis; Joint damage

\section{Related factors of juvenile idiopathic arthritis complicated with hyperuricemia}

Yazhen $\mathrm{Di}^{1}$, Ling $\mathrm{Wu}^{1}$, Yuanling Chen ${ }^{1}$, Jiapei Wang ${ }^{1}$, Mengjiao Shen $^{2}$, Shiwei Fu ${ }^{2}$

${ }^{1}$ Department of Pediatric Rheumatology and Immunology, Ningbo Women and Children's Hospital, 339 Liuting Street, Ningbo, Zhejiang 315000, China

${ }^{2}$ Medicine School of Ningbo University, 818 Fenghua Road, Ningbo, Zhejiang 315000, China, Department of Pediatric Rheumatology and Immunology, Ningbo Women and Children's Hospital, 339 Liuting Street, Ningbo, Zhejiang 315012, China

Objective: To observe the related factors of juvenile idiopathic arthritis (JIA) complicated with hyperuricemia.

Method: A retrospective study of the 60 JIA patients enrolled with follow-up visit for more than 1 year and performed a single factor analysis of the clinical data, lab data and special drug use of JIA with hyperuricemia (Group A), and without hyperuricemia (Group B).

Results: Comparison between the two groups: the differences in age, active sacroiliitis, BMI, systolic pressure, serum creatinine, salicylazosulfapyridine application showed a statistical significance $(\mathrm{P}<0.05)$; the opposite results in sex, disease course, high disease activity, diastolic pressure, serum albumin, alanine aminotransferase, aspartate transaminase, triglyceride, total cholesterol, low-density lipoprotein cholesterol, high-density lipoprotein cholesterol, glucose, urea nitrogen, NSAIDS application, systemic glucocorticoid application, MTX application, Tumor necrosis factor-a inhibitors application $(\mathrm{P}>0.05)$

Conclusion: JIA patients were obese, high systolic pressure and after salicylazosulfapyridine treatment will be more likely to accompany with hyperuricemia.

Keywords: Juvenile idiopathic arthritis; hyperuricemia; risk factor

\section{Primary immunodeficiency diseases of Chinese children in Guangdong area during a 10-year period}

\section{Hui-Qin Xiao, Hua-Song Zeng}

Women and Children's Medical Center, Guangzhou Medical University, China

Objective: This study aimed to describe the frequency and clinical spectrum of 126 patients with primary immunodeficiency diseases (PIDs) in Guangzhou Women and Children's Medical Center from China over the last 10 years.

Method: We retrospectively analysed the records of all the patients identified to have specific PID from January 2006 to December 2015. The diagnosed patients were classified according to guidelines of International Union of Immunological Society (IUIS) into nine different subgroups.

Results: In total, 126 children (88.9\% males) from 125 unrelated families met the criteria and were included. PIDs spectra were as follows:51 patients were diagnosed as "Predominant antibody deficiencies(40.5\%), "43(34.1\%) with "Combined immunodeficiencies," 15 (11.9\%) with "Congenital defects of phagocyte number, function, or both,"13(10.3\%) with "Combined immunodeficiencies with associated or syndromic features", $2(1.6 \%)$ with "diseases of immune dysregulation" and 2(1.6\%) with "Autoinflammatory disorders". Patients from Guangdong and Guangxi provinces accounted for $49.2 \%$ $(n=62)$. The patients aged from first day of life to 14 years old. The median age of onset at presentation was 2.5 months (range 1 day -96 months) and the median time to the diagnosis was 12 months (range $2-168$ months). Genetic analysis was performed in 97 patients (77\%). 57 different mutations were identified from 9 pathogenic genes in 64 unrelated patients. 4 novel mutations were detected. 26 carriers were identified from 21 families. Pneumonia was the most common infections $(\mathrm{n}=90,71.4 \%) .61$ patients had infections in two to four organs (48.4\%). Autoimmune haemolytic anaemia was the most common manifestation of autoimmunity. None of the patients in this study developed malignancy. 56 patients $(44.4 \%)$ received intravenous broad-spectrum antibiotics and 72 patients (57.1\%) received intravenous immunoglobulin (IVIG). Only one patient with ADASCID received an unrelated cord blood transplant (6/10 HLA matched donor) without pre-treatment conditioning. The percentage of donor chimerism was $7.5 \%$ on day +14 , and the patient died of respiratory failure caused by the pulmonary and intracranial infections on day +26 . In this cohort, 51 patients (40.4\%) were alive and 55 patients $(43.75 \%)$ died from severe infections.

Conclusion: We hope our study can reinforce awareness and education of medical stuff about PIDs, improve the diagnostic and therapeutic techniques, and which could improve the quality of life among patients with PIDs in the near future.

Keywords: primary immunodeficiency, clinical manifestation, genetic analysis 


\section{ADA-deficient SCID}

\section{Xiao Huiqin, Zeng Huasong}

Women and Children's Medical Center, Guangzhou Medical University, China

Clinical manifestation: A two-month-old female presented with a chief complaint of diarrhoea and fever. The infant had enlarged alar lymph nodes in inoculate side after inoculation with BCG vaccine. She had 1-month history of recurrent fever, chronic diarrhoea, pneumonia, urinary tract infection, decreased appetite and weight loss. There was no family history of immunodeficiency or death in early infancy.

Physical examination: On physical exam the patient was failure to thrive, associated with oral candidiasis, lymphadenopathy, moist rales of lung, perianal dermatitis and umbilical hernia

Laboratory examination: A complete blood count revealed a haemoglobin level of $92 \mathrm{~g} / \mathrm{L}$,a total white blood cell counts of $11 \times 10^{9} / \mathrm{L}$, a differential white blood cell counts of $70 \%$ neutrophils, $17 \%$ lymphocytes, and a platelet count of $317 \times 10^{9} /$ L.C-reactive protein was $123 \mathrm{mg} / \mathrm{L}$. The serum immunoglobulin test results showed in Table 1. The absolute lymphocyte count showed in Table 2 . The lymphocyte analysis showed T-B- $\mathrm{NK}^{+}$phenotype. The sputum culture was positive for Stenotrophomonas maltophilia. The chest CT revealed interstitial pneumonia.

Genetic test: Molecular studies on her blood DNA revealed the patient to be a compound heterozygote for 2 different mutations in ADA gene. One of which was a singlenucleotide substitution $(905 \mathrm{C} \rightarrow \mathrm{T})$ predicted to lead to a single amino acid substitution (S302F), the father was carrier (Figure 1). The second of which was a singlenucleotide duplication (747 dup $\mathrm{T}$ ) predicted to lead to a nonsense mutation (N250_R251 delins X) and the mother was carrier (Figure 2).

Treatment and outcome: ADA-SCID was diagnosed when the patient was 3 months of age. She was treated with multiple antibiotics, tuberculostearic drug, anti-fungal prophylaxis and immunoglobulin replacement therapy. Then she developed purulent meningitis. After changing anti-infective treatment, she received an unrelated cord blood transplant (6/10 HLA matched donor). The prevention of GVHD included CsA, MMF and tacrolimus. The percentage of donor chimerism was $7.5 \%$ on day +14 , and the patient died of respiratory failure caused by the pulmonary and intracranial infections on day +26 .

Keywords: ADA-SCID, CsA, tacrolimus

Table 1. The serum immunoglobulin test.

\begin{tabular}{|l|l|l|l|}
\hline $\operatorname{IgG}$ & $\operatorname{IgA}$ & $\operatorname{IgM}$ & $\operatorname{IgE}$ \\
$(3.22-7.18 \mathrm{~g} / \mathrm{L})$ & $(0.13-0.35 \mathrm{~g} / \mathrm{L})$ & $(0.23-0.91 \mathrm{~g} / \mathrm{L})$ & $(0-15 \mathrm{IU} / \mathrm{ml})$ \\
\hline 0.36 & $<0.07$ & 0.05 & $<5$ \\
\hline
\end{tabular}

Table 2. The absolute lymphocyte counts.

\begin{tabular}{|l|l|l|}
\hline CD3+T (690-2540cells/ul) & CD19+B (90-660cells/ul) & CD16+56+NK (90-590cells/ul)
\end{tabular} \begin{tabular}{l|l|l|}
\hline 18.83 & 2.79 & 48.22 \\
\hline
\end{tabular}

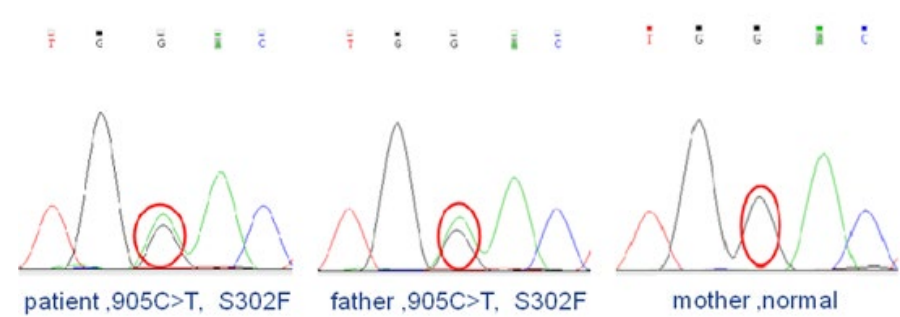

Figure 1. The genetic analysis of patient $\&$ father.

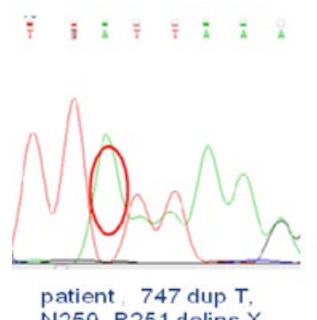

N250_R251 delins $X$

Figure 2. The genetic analysis of patient \& mother.

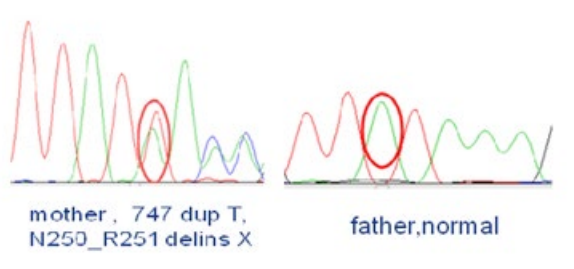

Study on the clinical effects of Bushen Formula on patients with chronic hepatitis $B$ treated by enticavir and the relevant immunoregulatory mechanism

Man Li, Long-Shan JI, Xin Zhang, Xue-Hua Sun, Yue-Qiu Gao

Laboratory of Cellular Immunity Shuguang Hospital Affiliated to TCM, Shanghai, China

Objective: To study the clinical effects of Bushen Formula on chronic hepatitis $\mathrm{B}$ patients $(\mathrm{CHB})$ treated by entecavir and the immunoregulatory mechanism of Bushen Formula.

Methods: Cohort study of clinical efficacy of Bushen Formula was proceeded. $100 \mathrm{CHB}$ patients treated by entecavir for 6 months or more were collected in outpatient of department of hepatopathy in Shuguang Hospital from February 2014 to December 2016. 100 CHB patients were categorized into the treatment group (52 patients) and the control group (48 patients). The patients in the treatment group received Bushen Formula combined with entecavir, and the patients in the control group continued with entecavir alone. The observation period was 24 weeks. The serum levels of ALT, AST, HBV DNA, HBsAg, HBeAg and the Chinese medicine symptom complex score were calculated.

$34 \mathrm{CHB}$ patients treated by entecavir for 6 months or more were collected from April 2015 to December 2016, and they were randomly categorized into the treatment group (15 patients) and the control group (19 patients). The observation period was 24 weeks. In addition, 9 healthy controls were enrolled. Serum ALT, AST, HBV DNA, HBsAg and HBeAg levels were tested. The peripheral frequencies of Th1, Treg, DC and the expression of PD-1/PD-L1 on T cells and DC were detected by flow cytometry.

Results: The results of cohort study of clinical efficacy of Bushen Formula after treatment showed that the Chinese medicine symptom complex score in both groups was decreased, and the score in treatment 
group was lower than that in control group $(\mathrm{P}<0.05)$; the change of HBsAg level in the treatment group was significantly more than that in the control group $(\mathrm{P}<0.05)$; the expression levels of HBV DNA and $\mathrm{HBeAg}$ in both groups were found statistically insignificant $(\mathrm{P}>0.05)$. The frequencies of Th1 and DC cells in CHB patients were significantly lower than those in healthy controls $(\mathrm{P}<0.05)$; the frequencies of Treg and $\mathrm{PD}-\mathrm{L} 1^{+}$Treg cells in $\mathrm{CHB}$ patients were higher than those in the healthy controls $(\mathrm{P}<0.05)$. The frequency of Th1 cell in the treatment group was significantly increased after treatment $(\mathrm{P}<0.05)$, while there was no significant change in the control group $(\mathrm{P}<0.05)$.

Conclusion: The clinical curative effects of Bushen formula combined with entecavir in $\mathrm{CHB}$ patients treated by entecavir were better than entecavir alone including the decrease of Chinese medicine symptom complex score and serum HBsAg. The immunoregulatory mechanism of Bushen Formula was related to the upregulation of the peripheral frequencies of Th1 and DC in CHB patients.

Keywords: Bushen formula, immunoregulatory, chronic hepatitis B

$A$ hybrid of $B$ and $T$ lymphoblastic cell line could potentially substitute dendritic cells to efficiently expand out Her-2/neu specific cytotoxic $T$ lymphocytes from breast cancer patients in vitro

\section{Sheng Chen ${ }^{1}$, Feifei $\mathrm{Gu}^{1}$, Kang $\mathrm{Li}^{1}$, Wei Gao ${ }^{2}$, Gang $\mathrm{Wu}^{1}$, Li Liu${ }^{1}$ \\ ${ }^{1}$ Cancer Center, Union Hospital, Tongji Medical College, Huazhong} University of Science and Technology, Wuhan, PR China

${ }^{2}$ Traumatology Department, Tongji Hospital, Tongji Medical College, Huazhong University of Science and Technology, Wuhan, PR China

Background: The ex vivo activation and expansion of antigen specific cytotoxic $\mathrm{T}$ lymphocytes (CTLs) has important meaning for antigen-recognition, antigen-epitope discovery and cytotoxicity research. Moreover, adoptive transfer of the expanded antigen specific CTLs holds promises to cure the cancer. However, all these advantages are hindered by lacking a robust way to specifically expand out CTLs.

Methods: We used a hybrid of B lymphoblastic cell line and T lymphoblastic cell line (T2 cells) as a substitute of dendritic cells (DCs), together with irradiated autologous PBMC as feeder cells and rhIL-2, to activate and expand Her-2/neu specific $\mathrm{CD}^{+} \mathrm{T}$ cells from Her-2/ neu and HLA-A2 double positive advanced breast cancer patients in vitro. The expansion index was counted by $\left[{ }^{3} \mathrm{H}\right]$ thymidine uptake, the specificity of the expanded Her-2/neu specific $\mathrm{CD}^{+} \mathrm{T}$ cells was verified by Her- $2 /$ neu conjugated HLA-A ${ }^{*} 0201$ Dextramer, and the cytotoxicity of expanded CTLs to breast cancer cell lines was analysed by ${ }^{51} \mathrm{Cr}$-release assay.

Results: These Her-2/neu loaded T2 cells could stimulate CD8 ${ }^{+} \mathrm{T}$ cells to secret IFN- $\gamma$ equally as DCs. With the addition of irradiated autologous PBMC as feeder cells and rhIL-2, they further expanded out Her-2/neu specific CD8 ${ }^{+} \mathrm{T}$ cells to $10^{7}$ in 8 weeks, nearly as a clinically used amount. Furthermore, these Her-2/neu specific CD8 ${ }^{+} \mathrm{T}$ cells had good sensitivity of recognition and killing Her-2/neu expressed breast cancer cell line SK.BR.3, but not the controls.

Conclusions: Her-2/neu loaded T2 cells could activate and expand out Her-2/neu specific CTLs equally as DCs. T2 cells might potentially substitute DCs to expand specific CTLs in vitro. This has important meaning in $\mathrm{T}$ cell immune function research, and also gives us another insight on how to rapidly obtain sufficient CTLs for adoptive cancer immunotherapy.
Keywords: Adoptive cancer immunotherapy, Hybrid of B and T lymphoblastic cell line, Cytotoxic T lymphocytes, Breast cancer

The proportion, origin and pro-inflammation roles of lowdensity neutrophils in SFTS disease

Huiyu Li ${ }^{1}$, Honglin Jin², Yajiao $\mathrm{Li}^{1}$, Hua Wang ${ }^{2}$, Hong Pan ${ }^{3}$, Huixia Zhao $^{2}$, Shenghua Jie ${ }^{2}$

${ }^{1}$ Union Hospital, Tongji Medical College, Huazhong University of Science and Technology, Wuhan, 430022, China

${ }^{2}$ Cancer Center, Union Hospital, Tongji Medical College, Huazhong University of Science and Technology, Wuhan, 430022, China

${ }^{3}$ Department of Infectious Diseases, Union Hospital, Tongji Medical College, Huazhong University of Science and Technology, Wuhan, 430022, China

${ }^{4}$ Kingstar global, Wuhan, 430072, China

Background: Severe fever with thrombocytopenia syndrome (SFTS) is an emerging viral infectious disease with a high case-fatality rate. We have preliminary findings on the existence of abnormal percentage of low-density neutrophils (LDNs) and normal density neutrophils (NDNs) in the circulating blood of SFTS patients. we further explored the percentage, origins and functional roles of LDNs in SFTS.

Methods: The LDNs and NDNs from circulating of SFTS or normal volunteers were purified separately. The percentage, origins and the phagocytic capability of SFTS viral (SFTSV) of LDNs were investigated by flow cytometry and real time PCR. The capacity of LDNs to secret of proinflammatory cytokines, anti-inflammatory cytokines and to damage endothelial cells were assessed by ELISA and flow cytometry.

Results: We observed that the proportion of LDNs increased dramatically compared with the healthy donors and became the dominant circulating neutrophil population in SFTS patients. Interestingly, the NDNs from the normal donors could switch to active LDNs under the SFTS environment. Moreover, SFTSV load in LDNs from the severe SFTS patients was significantly higher than that from the moderated SFTS patients. In addition, the LDNs secreted much higher levels of pro-inflamtory cytokines than NDNs in SFTS and induced endothelial cell injury.

Conclusion: The NDNs retained the developmental plasticity and could convert to LDNs, which contributed to the main sources of increased percentage of LDNs in SFTS. The LDNs exhibit proinflammation functions in SFTSV infection and may play pivotal roles in SFTS progress.

\section{Keywords: LDNs, conversion, NDNs, SFTS, SFTSV}

Down-regulation of notch signaling pathway reverses the Th1/Th2 imbalance in tuberculosis Patients

Qifeng Li ${ }^{1}$, Hui Zhang ${ }^{2}$, Liang Yu' ${ }^{1}$ Chao Wu ${ }^{3}$, Xinhui Luo ${ }^{1}$, He Sun ${ }^{1}$, Jianbing Ding ${ }^{4}$

${ }^{1}$ Xinjiang Institute of Paediatrics, People's Hospital of Xinjiang Uygur Autonomous Region, Urumqi 830001, Xinjiang, China

${ }^{2}$ Clinical Medicine Research Center, People's Hospital of Xinjiang Uygur Autonomous Region, Urumqi 830001, Xinjiang, China

${ }^{3}$ Department of Respiratory and Critical Care Medicine, People's Hospital of Xinjiang Uygur Autonomous Region, Urumqi 830001, Xinjiang, China 
${ }^{4}$ Department of Immunology, School of Preclinical Medicine, Xinjiang Medical University, Urumqi 830011, Xinjiang, China

Objective: Th1/Th2 imbalance to Th2 is of significance in the peripheral immune responses in Tuberculosis (TB) development. However, the imbalance mechanisms are still not well determined. Notch signalling pathway is involved in the peripheral $\mathrm{T}$ cell activation and effector cell differentiation. However, whether it affects Th1/Th2 imbalance in TB patients is still not known.

Methods: We used $\gamma$-secretase inhibitor (DAPT) to treat the peripheral blood mononuclear cells (PBMCs) from healthy people or individuals with latent or active TB infection in vitro, respectively. Then, the Th1/Th2 ratios were determined by flow cytometry, and IFN- $\gamma$, IL-4, IL-10 in the culture supernatant were measured by CBA method. The Notch signal pathway associated proteins Hes1, GATA3 and T-bet were quantitated by real-time PCR and Immunoblotting.

Results: Our results showed that DAPT effectively inhibited the mRNA and protein level of Hes1. In TB patients, the Th2 ratio increased in the PBMCs, alone with the high expression of GATA3 and IL-4, resulting in the high ratios of Th2/Th1 and GATA3/T-bet in TB patients. However, after blocking the Notch signalling pathway by DAPT, we found that the proportion of Th2 cells decreased, and the Th2/Th1 ratio in TB patients were DAPT dose-dependent, accompanied by the decrease of IL- 4 and GATA3. But, its influence on Th1 ratio and Th1 related T-bet and IFN- $\gamma$ levels were not significant.

Conclusions: Our results suggest that blocking Notch signalling by DAPT could inhibit Th2 responses and restore Th1/Th2 imbalance in TB patients.

Key-words: Notch signalling pathway, Th1/Th2 imbalance, Tuberculosis

The single nucleotide polymorphisms in TNF-a promoter are associated with susceptibility and clinical features of pulmonary tuberculosis in Chinese Uygurs

Qifeng $\mathrm{Li}^{1}$, Fengbo Zhang ${ }^{2}$, Yunjuan Zhao ${ }^{3}$, Xiangyan $\mathrm{He}^{4}$, Xiaoling Zhang $^{5}$, Guoqing Wang ${ }^{6}$, Fan $\mathrm{Li}^{6}$, Jianbing Ding ${ }^{3}$

${ }^{1}$ Xinjiang Institute of Paediatrics, People's Hospital of Xinjiang Uygur Autonomous Region, Urumqi 830001, Xinjiang, China

${ }^{2}$ Department of Clinical Laboratory, The First Affiliated Hospital of Xinjiang Medical University, Urumqi 830054, Xinjiang, China

${ }^{3}$ Department of Immunology, School of Preclinical Medicine, Xinjiang Medical University, Urumqi 830011, Xinjiang, China

${ }^{4}$ Party Committee Office, People's Hospital of Xinjiang Uygur Autonomous Region, Urumqi 830001, Xinjiang, China

${ }^{5}$ Clinical research Center, People's Hospital of Xinjiang Uygur Autonomous Region, Urumqi 830001, Xinjiang, China

${ }^{6}$ The Key Laboratory of Zoonosis, Department of Pathogenic Biology, College of Basic Medical Science of Jilin University, Changchun 130021, Jilin, China

Objective: Tuberculosis (TB) is a serious infectious disease, particularly in Chinese Uygurs. The tumour necrosis factor alpha (TNF- $\alpha$ ) that is involved in the interactions between the pathogens and host immune systems is highly related to TB susceptibility and diversity in different ethnic groups. The aim of this study is to investigate the relationship of TNF- $\alpha$ polymorphisms with pulmonary TB susceptibility in Chinese Uygurs.
Methods: This case-control study included 306 patients and 280 healthy controls. The characteristics of these participants were recorded and analysed. Five single nucleotide polymorphisms (SNPs) in TNF- $\alpha$ were genotyped by polymerase chain reaction-restriction fragment length polymorphism. Logistic regression was used to assess the association of the polymorphisms with TB susceptibility.

Results: Individuals with $\mathrm{BMI}<18.5 \mathrm{~kg} / \mathrm{m}^{2}$ or smoking were susceptible to TB. The frequencies of SNP rs 1800630 in the pulmonary TB patients were significantly different from those in healthy controls. In addition, the TNF- $\alpha$ SNP rs1799964 (OR=4.037, 95\% CI 2.4036.784) was associated with drug-resistance of TB. However, the polymorphisms of TNF- $\alpha$ were not related to conversion of sputum culture.

Conclusion: SNPs in TNF- $\alpha$ promoter are associated with susceptibility and drug-resistance of pulmonary TB in Chinese Uygurs. These TNF- $\alpha$ polymorphisms may be considered as risk factors for active pulmonary $\mathrm{TB}$.

Keywords: Tumour necrosis factor alpha (TNF- $\alpha$ ), Tuberculosis, Polymorphism, Susceptibility, single nucleotide polymorphisms (SNPs), Drug-resistance.

\section{Oncolytic herpes simplex virus type 2 induces potent immune response during the anti-tumor activity}

Wang Fei-Fei ${ }^{1,2,3}$, Guan $\mathbf{Q i}^{2,3}$

${ }^{1}$ Inner Mongolia University For the Nationalities, Tongliao 028000 China

${ }^{2}$ Inner Mongolia Forestry General Hospital, Yakeshi 022150 china

${ }^{3}$ The Second Clinical Medical School of Inner Mongolia University for the Nationalities, Yakeshi 022150 china

Oncolytic viruses are promising treatments for many kinds of solid tumors. Oncolytic herpes simplex virus type 2 (oHSV2) is an oncolytic agent that encodes granulocyte-macrophage colony-stimulating factor and is genetically engineered to selectively replicate in and kill tumor cells, while sparing normal cells. In vitro, oHSV2 could infect most of the human and murine tumor cell lines and was highly oncolytic. Initial oHSV2 infection, cell necrosis, and subsequent oHSV2 propagation within the tumor cell led to a cascade of host responses, reflective of natural anti-viral immune responses. Although anti-viral immune responses limited oHSV2, induced the anti-tumor immunity. There was a study showed that oHSV2 treatment not only slowed down the growth of tumors without causing weight loss but also induced an elevation of NK cells and mild decrease of Tregs in spleen. Another study also found oHSV2 was able to reduce the ratio of spleen MDSC and Tregs in the experimental mouse, simultaneously increased the number of mature dendritic cells in tumor-draining lymph nodes and the effective $\mathrm{CD}^{+} \mathrm{T}$ and $\mathrm{CD} 8^{+} \mathrm{T}$ cells in the tumor microenvironment. In addition, oHSV2 could significantly prolong the survival of tumor-bearing mice. Therefore, we concluded that oHSV2 is an effective therapeutic cancer candidate that induces an oncolytic effect and recruits adaptive immune responses for an enhanced therapeutic impact, may be a safe and effective therapeutic agent for cancer treatment.

Keywords: Oncolytic herpes simplex virus type 2 (oHSV2), Dendritic cells (DC), Immune response, Micro-environment 
Role of microRNA-29b-3p in Schistosoma japonicuminduced hepatic fibrosis by targeting COL1A1 and COL3A1: An in vivo and vitro study

Hongyan Kong ${ }^{1}$, Ran Tao ${ }^{1}$, Xiangxue Fan ${ }^{2}$, Hongyue Zhang ${ }^{1}$, Qiqing Song1, Yu Huang ${ }^{3}$, Jiaquan Huang ${ }^{1}$

${ }^{1}$ Department of Infectious Disease, Tongji Hospital, Tongji Medical College of Huazhong University of Science and Technology, Wuhan 430030, P.R. China

${ }^{2}$ Department of Infectious Disease, Liaocheng People’s Hospital, Liaocheng 252000, P.R. China

${ }^{3}$ Department of Pediatrics, Tongji Hospital, Tongji Medical College of Huazhong University of Science and Technology, Wuhan 430030, P.R. China

Aim: This study aimed at identifying the in vivo and vitro effects of microRNA-29b-3p (miR-29b-3p) in Schistosoma japonicum (S. japonicum)-induced hepatic fibrosis by targeting COL1A1 and COL3A1.

Methods: Thirty male Balb/c mice were divided on random into the blank group and the model group. The mice in the model group were percutaneously infected with cercariae of S. japonicum. NIH3T3 mouse embryonic fibroblasts were obtained and designated into 6 groups: the blank, negative control (NC), miR-29b-3p mimic, TGF- $\beta 1$ ( $10 \mathrm{ng} / \mathrm{ml})$, TGF- $\beta 1+\mathrm{NC}$, and TGF- $\beta 1+$ miR-29b-3p mimic groups. To identify the relationships of mir-29b-3p with COL1A1 and COL3A1 dual luciferase reporter gene was conducted. The qRTPCR was conducted to determine the expression of miR-29b-3p, COL1A1 mRNA and COL3A1 mRNA in hepatic tissues and NIH-3T3 fibroblasts. Western blotting and immunofluorescence staining were performed for observation of protein expressions of TIMP-1, HSP47, a-SMA, MMP-9, COL1A1 and COL3A1. For observing the viability and apoptosis of NIH-3T3 fibroblasts were CCK-8 assay and flow cytometry were conducted.

Results: The relative expression of miR-29b-3p was significantly down-regulated in hepatic tissues of mice of the model group. MiR-29b$3 p$ could directly target COL1A1 and COL $3 \mathrm{~A} 1$ genes. In comparison to the blank, NC, TGF- $\beta 1$ and TGF- $\beta 1+$ NC groups, NIH-3T3 fibroblasts exhibited miR-29b-3p and MMP-9 up-regulation in the miR-29b$3 p$ mimic group, while the expressions of TIMP-1, HSP47, $a$-SMA, COL1A1 and COL3A1 were obviously down-regulated. Furthermore, in comparison to the blank, NC, TGF- $\beta 1$ and TGF- $\beta 1+\mathrm{NC}$ groups, $\mathrm{NIH}-3 \mathrm{~T} 3$ fibroblasts in miR-29b-3p mimic group depictedlower cell viability and a higher apoptosis rate.

Conclusion: Together these data provide a strong evidence that miR-29b-3p could relieve $S$. japonicum-induced hepatic fibrosis by down-regulation of COL1A1 and COL3A1.

Keywords: Schistosoma japonicum; MicroRNA-29b-3p; COL1A1; COL3A1; Hepatic fibrosis

\section{BIN1 reverses immune escape via c-MYC/PD-L1 pathway in} non-small cell lung cancer

\section{Liu Lihua, Wang Jiali}

Department of Tumor Immuno-Therapy, Fourth Hospital of Hebei Medical University, Shi Jiazhuang 050035

Objective: Identifying the effect of BIN1 on the expression of PDL1 of NSCLC by gene transfection and siRNA methods and exploring the associated mechanisms.
Methods: qRT-PCR and Western blot assays were used to evaluate BIN1 expression levels in four NSCLC cell lines of H1975, H1650, A549, H460 and the embryo lung cell line 2BS. H1975 cells which express lowest level of BIN1 were transfected with BIN1 lentiviral vector $\left(\mathrm{BIN}^{+}\right)$or empty vector plasmid (pCDH). In addition, $\mathrm{H} 1975$ cells was performed as the Con group. qRT-PCR and Western blot assays were used to detect the effect of BIN1 transfection and the changes of PD-L1 expression. BIN1-siRNA and NC-siRNA plasmid were transfected into H460 cells. qRT-PCR and Western blot assays were used to detect the effects of RNA silencing of BIN1 and the effect of BIN1 low expression on PD-L1 expression. Immunofluorescence assay was used to detect the effect of BIN1 overexpression on c-MYC expression. c-MYCsiRNA and NC-siRNA were transfected into H1975 cells to confirm whether c-MYC was involved in the mechanisms of PD-L1 regulation.

Results: qRT-PCR and Western blot analysis showed the highestlevel expression of BIN1 in H460 cells compared to H1975, H1650, A549 and 2BS and the lowest level expression of BIN1 in H1975 cells compared to H1650, A549, H460 and 2BS $(P<0.05)$. The expression of BIN1 mRNA and protein in BIN1-overexpressed H1975 cells increased significantly compared with $\mathrm{pCDH}$ group and Con group $(P<0.05)$. An analysis of PD-L1 expression in BIN1-overexpressed H1975 cells revealed significantly decreased expression of PD-L1 with qRT-PCR and Western blot when compared to pCDH group and Con group $(P<0.05)$. Knock-down of BIN1 caused a significant increase in expression of PD-L1 compared to the Con group $(P<0.05)$. Immunofluorescence assay showed a significant decrease in expression of c-MYC in BIN1-overexpressed H1975 cells compared to pCDH group and Con group $(P<0.05)$. iRNA knockdown of c-MYC caused a reduction in the level of $\mathrm{PD}-\mathrm{L} 1 \mathrm{mRNA}$ and protein compared to the Con group $(P<0.05)$.

Conclusion: BIN1 could reverse the PD-L1-mediated immune escape via inhibiting c-MYC pathway in NSCLC.

Keywords: NSCLC, BIN1, PD-L1, c-MYC

Association of interleukin-10 gene single nucleotide polymorphisms with susceptibility to systemic lupus erythematosus in a Chinese population

Tian-Tian $\mathrm{Lv}^{1,2}$, Jun $\mathrm{Wu}^{1,2}$, Jun $\mathrm{Li}^{1,2}$, Tian-Ping Zhang ${ }^{1,2}$, Xiao-Ke Yang $^{1,2}$, Nan Xiang ${ }^{3}$, Yin-Guang Fan ${ }^{1,2}$, Hai-Feng Pan ${ }^{1,2}$, Bin Wang ${ }^{1,2}$

${ }^{1}$ Department of Epidemiology and Biostatistics, School of Public Health, Anhui Medical University, 81 Meishan Road, Hefei, Anhui, China

${ }^{2}$ Anhui Province Key Laboratory of Major Autoimmune Diseases, 81 Meishan Road, Hefei, Anhui, China

${ }^{3}$ Department of Rheumatology and Immunology, Anhui Provincial Hospital affiliated with Anhui Medical University, 17 Lujiang Road, Hefei, Anhui, China

Objective: The aim of this study was to investigate the association of interleukin (IL)-10 gene single nucleotide polymorphisms (SNPs) with susceptibility to systemic lupus erythematosus (SLE) in a Chinese population.

Methods: 787 SLE patients and 461 normal controls were recruited in this study. Nine SNPs in IL-10 gene (rs1518110, rs1518111, rs1554286, rs1800890, rs1800893, rs3024493, rs3024495, rs3024498 and rs6667202) were genotyped using TaqMan genotyping assays on Fluidigm 192.24 system. 
Results: Significant difference in genotype distribution of rs1800893 were detected between SLE patients and control subjects [TT versus CC: adjusted $\mathrm{OR}=3.421$ (95\% $\mathrm{CI}=1.127-10.386), \mathrm{P}=0.030$ ]. In addition, significant difference was also found in the recessive model (TT versus TC + CC: adjusted OR $=3.483$, $95 \% \mathrm{CI}=1.148$ $10.566, \mathrm{P}=0.028$ ), whereas no significant difference was discovered in the dominant model (TT + TC versus CC: adjusted $\mathrm{OR}=1.020,95 \%$ $\mathrm{CI}=0.729-1.428, \mathrm{P}=0.908)$. Besides, rs3024493 A allele and rs3024495 $\mathrm{T}$ allele were risk alleles of SLE ( $\mathrm{rs} 3024493$ : $\mathrm{OR}=1.652,95 \% \mathrm{CI}=1.047$ 2.606, $\mathrm{P}=0.031$; rs3024495: $\mathrm{OR}=1.628,95 \% \mathrm{CI}=1.031-2.571, \mathrm{P}=0.037)$. The frequency of IL-10 rs3024498C allele was significantly higher in-patient group compared with control subjects ( $\mathrm{OR}=5.525,95 \%$ $\mathrm{CI}=1.963-15.550, \mathrm{P}=0.001)$. Significant difference was also observed under the dominant model $(\mathrm{CC}+\mathrm{CT}$ versus TT: adjusted $\mathrm{OR}=0.231$, 95\% CI $=0.077-0.690, \mathrm{P}=0.009)$. Interestingly, significant differences were detected both in the allele and genotype frequencies of rs3024498 between SLE patients with and without arthritis $(\mathrm{P}=0.004, \mathrm{P}=0.025$, respectively). There was significant difference in genotype frequency at rs3024498 between SLE patients with and without malar rash $(\mathrm{P}=0.042)$. However, we failed to find any significant results between the groups with respect to rs1518110, rs1518111, rs1554286, rs1800890 and rs6667202 (all $\mathrm{P}>0.05$ ).

Conclusion: In summary, IL-10 rs3024498 polymorphism might contribute to SLE susceptibility and several clinical phenotypes.

Keywords: Systemic lupus erythematosus; Interleukin (IL)-10 gene; Single nucleotide polymorphisms

\section{The Expression of ETAR in Liver Cirrhosis and Liver Cancer}

Ran Tao ${ }^{1}$, Yu Huang ${ }^{2}$, Xiangxue Fan ${ }^{3}$, Hongyue Zhang', Hongyan Kong $^{1}$, Qiqing Song ${ }^{1}$, Jiaquan Huang ${ }^{1}$

${ }^{1}$ Department and Institute of Infectious Disease, Tongji Hospital, Tongji Medical College, Huazhong University of Science and Technology, Wuhan, 430077, Hubei, China

${ }^{2}$ Department of Endocrinology Affiliated Liyuan Hospital, Tongji Medical College, Huazhong University of Science and Technology, Wuhan, 430077, Hubei, China

${ }^{3}$ Department of Nephrology, The People's Hospital of Three Gorges University, Yichang, 443000, Hubei, China, 4 Department and Institute of Infectious Disease, Liaocheng People's Hospital, Liaocheng, 252000, Shandong, China

Background: To investigate the expression of endothelin receptors in liver diseases and discuss its role in the process of liver cirrhosis and liver cancer.

Research design and methods: We examined the expressions of ETAR, ETBR and $\alpha$-SMA in tissue samples using western blotting analysis. Furthermore, immunofluorescence was used to locate ETAR expression in hepatic stellate cells (HSCs) and hepatic sinusoidal endothelial cells (HSECs), we calculated the percentage of positive cells and then analysed its relationship with clinical indexes.

Results: According to the western blotting analysis, the expression of ETAR was high in hepatic hemangioma and liver cancer tissues and ETBR was highly expressed in cirrhosis tissues. The immune fluorescence results demonstrated that the expression of ETAR was elevated in hepatic hemangioma and liver cancer tissues. Moreover, ETAR expression was found in both HSCs and HSECs. Finally, the statistical analysis revealed that the number of positive ETAR cells was correlated with the clinical index platelets (PLT), alanine transaminase (ALT) and diameter of portal vein.

Conclusion: Endothelin receptors express differently in liver cirrhosis and liver cancer tissues and play a role in hepatic diseases by affecting HSCs and HSECs.

Keywords: ETAR, liver cirrhosis, HSCs and HSECs, liver cancer

\section{The effects of interleukin-24 on the cytotoxic activity of CIK cells to HepG2}

Wang Zhihua, Yuan Yutao, Huang Sunhui, Cui yali

Cancer Research Institute of Harbin Medical University, Harbin, China

Objective: To find a new method to enhance the cytotoxic activity of cytokine-induced killer (CIK) cells for clinical immunotherapy.

Methods: Mononuclear cells were extracted from the peripheral blood of health adults. One group was treated with interferon- $\gamma($ IFN- $\gamma$ ) at the 1st day, and interleukin-1 (IL-1), CD3mAb and IL-2 were added at the 2 nd day. And the other group was disposed with IL-24 besides IL-1, CD3mAb and IL-2. Cell counting method was used to determine the proliferation of CIK cells, and the cytotoxic activity was detected by MTT assay. Cell phenotype was examined by flow cytometry. The scanning electron microscopy and transmission electron microscopy were used to observe the killing effects of CIK cells on tumour cells as well as the changes of tumour cells.

Results: The proliferation of CIK cells without IL-24 treatment was higher than that with IL-24 treatment $(126.34 \pm 2.14$ vs $108.87 \pm 1.29$, $\mathrm{p}<0.05)$. The cytotoxic activities of CIK cells co-cultured with IL-24 were above $90 \%$ at different ratio of effect to target (10:1, 20:1, 40:1), which were significantly higher than those in the other groups $(10: 1$ : $95.58 \% \pm 2.21 \%$ vs $27.31 \% \pm 2.69 \%, 8.74 \% \pm 2.41 \%, 38.65 \% \pm 21.30 \%$, all $\mathrm{p}<0.05 ; 20: 1: 91.97 \% \pm 4.21 \%$ vs $34.27 \% \pm 0.85 \%, 11.54 \% \pm 2.78 \%$, $48.32 \% \pm 11.72 \%$, all $\mathrm{p}<0.05 ; 40: 1: 91.84 \% \pm 9.28 \%$ vs $50.67 \% \pm 1.30 \%$, $23.73 \% \pm 11.07 \%, 52.89 \% \pm 12.26 \%$, all $\mathrm{p}<0.05$ ). Cell phenotypes were not significantly different between the CIK cells with and without IL24 treatment $(\mathrm{p}>0.05)$. Apoptotic and necrotic cells were obviously increased in after IL-24 treatment under transmission electron microscope.

Conclusion: IL-24 can obviously enhance the cytotoxic activity of CIK cell.

Keywords: cytokine-induced killer cell; interleukin-24; cytotoxic activity

The basic and Clinical Trial progress of Natural killer (NK)
cell Wang Zhihua

${ }^{1}$ Nanjing Qingan Hospital, Nanjing, Jiangsu Province, 210017, China

${ }^{2}$ Cancer Institute, Haerbin Medical Universite, Haerbin, Heilongjiang Province, 150040, China

The NK cells as a unique immune effector cells in adoptive immunotherapy (AIT) have substantial proliferation in the short term, regulate the body's immunity, antitumor, antibacterial, antiviral, antiaging characteristics widely attention, clinical application and shows great potential and broad prospects. This article is only at home and abroad about the biological activity of NK cells, immune regulation and clinical application research status and progress are reviewed. 
Will also be NK cells and immune response, the relationship of NK cells is discussed and anti-aging, autism, epilepsy, aplastic anaemia, diabetes, lupus erythematosus (sle), and the NK cell immunotherapy was prospects forecasted too in this paper.

Keywords: immune treatment; NK cells; Clinical application

Strain and age shape the function of bone marrow-derived dendritic cells (BMDCs) in mice

Min Wu, Zhaoqi Lu, Liuchuang Gao, Miao He, Junyan Han

Department of Immunology, HuaZhong University of Science and Technology, Wuhan 430030, China

Objective: We aimed to investigate the influence of strain and age on the phenotype, function and apoptosis of BMDCs.

Methods: BMDCs were cultured in vitro from different strains (BALB/c and C57BL/6) and ages (1w, 3w and 8w). ELISA and Flow cytometry were performed to test the yield, purity, surface marker, cytokines expression level, apoptosis as well as the capacity of BMDCs to induce allogeneic $\mathrm{T}$ cells proliferation before and after LPS stimulation.

Results: 1 . The yield of BMDCs from C57BL/6 mice was higher than that from $B A L B / c$ mice $(p<0.05)$ in every age group, but there was no difference between age groups in the same strain. The purity of BMDCs from all the groups showed no significant difference $(p>0.05)$.

2. BMDCs from C57BL/6 were more matured than BALB/c in every age group as showed by the elevated percentage of $\mathrm{MHCII}+\mathrm{CD} 80+$ or CD86+ BMDCs $(\mathrm{p}<0.01)$. LPS stimulation promoted the maturation of BMDCs in all the groups ( $\mathrm{p}<0.01)$, except for the $\mathrm{BALB} / \mathrm{c}$ neonates, which remained unchanged ( $p>0.05)$. In the same strain, BMDCs of neonates were significantly less matured than BMDCs from $3 \mathrm{w}$ or $8 \mathrm{w}$ $(\mathrm{p}<0.01)$.

3. At baseline, BMDCs secreted no IL-6, IL-12 and TNF- $\alpha$. LPS stimulation resulted in increased production of TNF- $\alpha$ in both C57BL/6 and $B A L B / c$ mice $(p<0.01)$ of each age group. However, TNF- $\alpha$ secreted by $\mathrm{C} 57 \mathrm{BL} / 6$ was much higher than $\mathrm{BALB} / \mathrm{c}$ of same age $(\mathrm{p}<0.01)$. In the same strain, the level of TNF- $\alpha$ in neonates was significantly lower than other age groups $(\mathrm{p}<0.01)$. The secretion of IL- 6 and IL- 12 was similar to TNF- $\alpha$. However, BMDCs from BALB/c neonatal mice produced lower level of IL-12 before and after LPS stimulation.

4. BMDCs from $3 \mathrm{w}$ and $8 \mathrm{w}$ mice showed increased ability to induce allogeneic $\mathrm{T}$ cell proliferation compared with neonatal mice before and after LPS stimulation $(\mathrm{p}<0.01)$ in both strains. LPS stimulation significantly increased this ability except for $\mathrm{BALB} / \mathrm{c}$ neonates, which showed no difference.

5. In the same age group, the apoptosis of BMDCs in C57BL/6 mice was lower than that in BALB/c mice before and after LPS stimulation $(\mathrm{p}<0.01)$. LPS stimulation promoted BMDCs apoptosis in all the groups $(\mathrm{p}<0.05)$, especially in $\mathrm{BALB} / \mathrm{c}$ neonatal mice (up to $70 \%, \mathrm{p}<0.01$ ).

Conclusions: We found that the function of BMDCs from different strains and ages of mice is different. BMDCs from neonates were less mature and less sensitive to LPS stimulation. These results suggested that strain and age of mice could shape the immune responses via their effects on DCs.

Keywords: BMDCs, LPS, strain, age, neonate

\section{The intestinal microbiota in chronic kidney Disease}

Yu Jia-li' ${ }^{1}$, Da Jing-jing ${ }^{2}$, Dong Rong ${ }^{2}$, Yuan Jing ${ }^{2}$, Zuo Li ${ }^{1}$, Zha Yan ${ }^{1,2}$

${ }^{1}$ Department of Immunology, Guizhou Medical University, Guizhou 550004, China

${ }^{2}$ Institute of Nephritic and Urinary Disease, Guizhou Provincial People's Hospital, Guizhou 550002, China

The intestinal microbiota plays a central role in certain physiological functions that contribute to the maintenance of symbiotic relationship with human hosts, such as metabolism, nutrition, entero protection and immunoregulation function, etc. Nevertheless, recent studies have revealed that the alerted gut microbiome in patients with chronic kidney disease (CKD) results in exacerbating the progression of CKD and CKD-related complications, which is distinct from the role of symbiotic microbiota. This paper is intended to provide an overview of the connections between intestinal flora and CKD. In addition, the main mechanisms and the potential intervention strategy are also carried on the review and discussed.

Keywords: Intestinal microbiota; Chronic kidney disease; CKDrelated complications

The characteristics analysis of intestinal guts features in cerebral infarction and coronary heart disease patients

\section{Yu Zhu}

Department of Clinical Laboratory, Tianjin Huanhu Hospital, Tianjin Key Laboratory of Cerebral Vessels and Neural Degeneration, Tianjin, 300350 China

Objective: To, investigate to the abundance and characteristic of intestinal flora of cerebral infarction (CI) and coronary heart disease (CHD) patients.

Method: 10 fecal samples from patients with cerebral infarction and coronary heart disease and 10 samples from healthy volunteers as control were selected to determine the abundance of intestinal flora using sequencing technology for characteristic analysis.

Results: Compared with health group, the Bacteroidetes, et al. was decreased and Firmicutes, et al. was increased in CI group, the Proteobacteria, et al. was decreased and Firmicutes, et al. was increased in CHD group in phylum, meanwhile, the Bacteroides, et al. was decreased and Escherichia-Shigella, et al. was increased in CI group, the Escherichia-Shigella, et al. was decreased and Megamonas, et al. was increased in CHD group in genus, the flora composition was low similarity in CI and CHD group, we also analysed the evolutionary branch and intercommunication in CI and CHD group.

Conclusion: There was a confusion and low similarity in intestinal guts of $\mathrm{CI}$ and $\mathrm{CHD}$, and intestinal guts may be a potential marker of above disease.

Keywords: cerebral infarction, coronary heart disease, intestinal guts

Genetic analysis retrospectively in 1303 cases with beta thalassaemia children

Li Wanling, Hua Liang, Tang Ying, Zhu Bing, Han Ning, Kuang Lu, Huasong Zeng

Central Laboratory, Guangzhou Women and Children's Medical Center, Guangzhou Medical University Guangzhou, Guangdong 510120, China 
Objective: To understand the detection rate and gene phenotypic characteristics of high-risk children in Guangzhou.

Methods: 2ml (EDTA anticoagulant) of blood were collected from 6171 cases with high-risk thalassemia children. The gene mutation type of children was determined by PCR and reverse point hybridization.

Results: $1303 \beta$-thalassemia cases were detected in 6171 cases (1303/6171,21.11\%). 17 kinds of gene mutation detection and 35 genotypes were detected. The most common 7 kinds of $\beta$-thalassemia gene mutations were CD41-42, IVS-2-654, -28, CD17, CD71-72, beta $\mathrm{E}$, and CD43, which accounting for $38.83 \%, 27.24 \%, 9.82 \%, 9.06 \%$, $2.23 \%, 2.15 \%, 1.15 \%$,respectively. These 7 kinds of gene mutations contributed to $90.48 \%$ of all mutated genes. 1218 cases of heterozygote accounting for $93.48 \%, 32$ cases of homozygous (2.46\%) and 53 cases (4.06\%) double heterozygote were detected.

Conclusion: The type of beta thalassemia gene mutations in children was diversity in Guangzhou region.

The main type of $\beta$-thalassemia is heterozygous. It is a great significance to reduce the birth of serious thalassemia children through strengthen the premarital and pregnancy thalassaemia screening and prenatal diagnosis.

Keywords: Beta-thalassaemia; Gene analysis; children

\section{The effect of varicella-zoster virus on the immune system of patients with systemic lupus erythematosus}

\section{Cui Yongwang, Zeng Huasong}

Department of Allergy, Immunology and Rheumatology, Guangzhou Women and Children's Medical Center, Guangzhou Children's Hospital, Guangzhou Medical University, China

Objective: To observe the immunological indexes' changes of patients with chickenpox, systemic lupus erythematosus and systemic lupus erythematosus with chickenpox, and to explore the effect of varicella-zoster virus on the immune system of patients with systemic lupus erythematosus.

Methods: A total of sixty-six cases of patients with chickenpox, systemic lupus erythematosus and systemic lupus erythematosus with chickenpox were choose, and Diagnosis and treatment of chickenpox in our hospital patients, in with systemic lupus erythematosus and systemic lupus erythematosus chickenpox patients, twenty-one cases of normal control group were set at the same time; The white blood cell count (WBC), platelet count (PLT) and haemoglobin content (HGB) in every group were detected using blood cells analyser; The levels of immunoglobulin G (IgG), immunoglobulin A (IgA) and immunoglobulin $\mathrm{M}$ (IgM) and C3, C4 in serum of every group were detected using immunoturbidimetric assay method.

Results: Compared with the control group, the levels of WBC, PLT and HGB in groups of varicella, SLE and SLE with varicella were decreased, and the reduction of WBC and PLT in group of SLE and SLE with varicella had statistical significance $(\mathrm{P}<0.05$ or $\mathrm{P}<0.01)$; The content of IgG, IgA and IgM in serum of varicella group decreased while the group of SLE and SLE with varicella increased, and the reduction of IgA in serum of varicella group had statistical significance $(\mathrm{P}<0.05)$ while the increase of IgG and IgA in group of SLE and SLE with varicella had statistical significance $(\mathrm{P}<0.05$ or $\mathrm{P}<0.01)$; The levels of $\mathrm{C} 3$ and $\mathrm{C} 4$ in serum of varicella group increased while the group of SLE and SLE with varicella decreased, and the reduction of $\mathrm{C} 3$ and $\mathrm{C} 4$ in group of SLE with had statistical significance $(\mathrm{P}<0.05)$.
Compared with the SLE group, the increase of WBC had statistical significance $(\mathrm{P}<0.05)$ in SLE with varicella while the levels of IgG, IgA, $\mathrm{C} 3$ and $\mathrm{C} 4$ in serum decreased with statistical significance $(\mathrm{P}<0.05)$.

Conclusion: Varicella-zoster virus can induce the changes of immune system-related indexes in systemic lupus erythematosus patients.

Keywords: Varicella-zoster virus; Systemic lupus erythematosus; Immune system

The clinical features and perforin A91V gene analysis in 31 macrophage activation syndromes with systemic onset juvenile idiopathic arthritis cases in China

Wei Ping, Chen Xiangyuan, Chen Huishan, Wei Yandan, Zeng Ping, Xie Ying, Li Feng, Tang Ying, Zeng Huasong

Department of Allergy, Immunology and Rheumatology, Guangzhou Children's Hospital, Guangzhou Women and Children's Medical Center, Guangzhou Medical College, Guangzhou 510120, Guangdong, China

Objectives: Macrophage activation syndrome (MAS) is a severe, potentially fatal complication of rheumatoid disease, especially in the systemic onset juvenile idiopathic arthritis (SoJIA). We investigated the clinical characteristics of 31 Chinese MAS cases with SoJIA and detected the perforin A91V gene in part cases.

Methods: Clinical characteristics of 31 SoJIA with MAS cases in the last nine years in our institute were retrospectively analysed. Genespecific polymerase chain reaction (PCR) primers were used to analyse the perforin A91Vgene polymorphism.

Results: 31 SoJIA cases were associated with MAS. No specific medication was identified as trigger. $83 \%$ cases had infections prior to MAS. Clinical manifestations of MAS included persistent fever (100 $\%)$, hepatosplenomegaly (93.55\%), lymphadenopathy (64.52\%), and liver dysfunction (83.87\%). Laboratory features included pancytopenia (41.9\%), increased serum ferritin $(87.10 \%)$, triglycerides $(74.19 \%)$ and lactic dehydrogenase (87.10\%). Hemophagocytosis were found in 27 cases $(87.10 \%)$. The levels of NKcell in MAS patients was statistically below than the SoJIA and control group. The perforin A91V variant gene was detected in twenty cases, but no mutation was found. Glucocorticoid, intravenous immunoglobulin, immunoimpressive therapy were effective. After treatment, 28cases (90.32\%) were in remission, while 3 out of 31 cases died with a mortality of $9.68 \%$.

Conclusions: MAS is a life-threatening complication of SoJIA. Most cases were preceded by infection. Unremitted fever, progressive hepatosplenomegaly, lymphadenopathy, cytopenias, elevated serum liver enzymes, significantly increased serum ferritin are the main feature. Prompt recognition and treatment is the key to improve prognosis. The perforin gene mutations in our patients need more research. The numbers of the cases need expend.

Keywords: Perforin, Systemic onset juvenile idiopathic arthritis, Macrophage activation syndrome, NK cells.

Copyright: (C2019 Zeng H. This is an open-access article distributed under the terms of the Creative Commons Attribution License, which permits unrestricted use, distribution, and reproduction in any medium, provided the original author and source are credited. 